\title{
Unde Exoriar? En los cien años del "Curso de lingüística general" de Ferdinand de Saussure
}

\section{Unde Exoriar? In the one hundred years of the "Course on General Linguistics" by Ferdinand de Saussure}

\author{
Miguel Ángel Mahecha B. \\ Universidad Surcolombiana, Colombia
}

\begin{abstract}
Mais Saussure est avant tout un linguiste: c'est d'une pratique grammaticale sur des langues qu'il tire son expérience et les fondements de sa réflexion sur le linguistique.
\end{abstract}

Gadet, 1990, 7

\section{Proemio}

En el número 16 de la revista Forma y Función (2003, pp. 310-323) publicamos una reseña del libro Ecrits de linguistique générale [ELG] de Ferdinand de Saussure. En cierta forma la reseña tenía un matiz apologético e invitaba a una lectura diferente de la obra del ginebrino tomando distancia del Curso de lingüística general [CLG], publicado por primera vez en $1916 \mathrm{y}$ destacando además que dicho libro no había sido escrito por Ferdinand de Saussure. La novedad que aparentemente ofrecían los ELG se apoyaba en la idea [excluyente y polarizada] de que constituían de manera exclusiva el corpus auténtico y original de los propios escritos de Saussure sobre la lingüística general. Al volver a la reseña después de trece años, entendemos por qué la primera parte (pp. 310-315) se consagró -y en ese momento no se pensó así- al CLG: su existencia validaba los ELG y no al contrario como podía creerse (cf. Trabant, 2005). Y una manera de entender los Escritos es considerando las particularidades editoriales del Curso. No es el único camino pero vale la pena recorrerlo.

Esta presentación retoma y reelabora algunos elementos de la reseña citada, comenta brevemente los estudios del dossier y sugiere una lectura de los diferentes textos de Saussure incluyendo por supuesto el CLG en las aulas universitarias teniendo en cuenta un corpus intertextual que no ha sido estudiado en nuestro contexto colombiano ${ }^{1}$.

El orden de la presentación es el siguiente. En primer lugar se retoma la primera parte de la reseña del año 2003 ocupada en describir el CLG. Enseguida se describen algunos detalles de cómo fue escrito el CLG (segunda parte), se menciona la presencia e influencia de Saussure en el mundo hispano (tercera parte) para finalizar en el comentario de las contribuciones al dossier (cuarta parte).

1 En su reciente libro Saussure au futur (2015, reseñado aquí), el profesor Rastier propone un nuevo acercamiento al pensamiento de Saussure haciendo una lectura crítica, hermenéutica e integradora del gran corpus textual que se ha ido encontrando y estudiando con el paso del tiempo [empezando por las fuentes manuscritas de Robert Godel (1957)] a fin de dimensionar en su justa medida su aporte a la consolidación de una teoría lingüística: "Este libro no se propone participar en la iconización de Saussure, pero enfatiza la necesidad de investigaciones filológicas, de encuestas hermenéuticas y de reflexiones lingüísticas para restituir el proyecto saussureano. En efecto, poder caracterizar y comprender una obra reconocida como fundadora es, evidentemente, una apuesta por el porvenir de la lingüística [...] Paradojas de un corpus extendido y de una lectura atrasada, los textos publicados en 2002, especialmente los inéditos del Invernadero, pertenecen a nuestro siglo. A nosotros nos corresponde leerlos, interpretarlos, valorar su alcance con respecto a la fecha de su redacción (verosímilmente la década de 1890) como en relación al corpus saussureano, a la historia misma del saussurismo y al porvenir de la lingüística." [pp. 7 y 10, versión española de Enrique Ballón Aguirre, 2016]. Como complemento a esta obra, contamos con el valioso estudio de la profesora colombiana Claudia Mejía (especialista colombiana en la vida y obra de Saussure) publicado en 2015 y que presenta en detalle un inventario de manuscritos de Saussure (manuscrits de Vufflens). 
en la vulgata, atribuyéndosela sólo a de Saussure y con frecuencia se le acusa injustamente por el limitado tratamiento que le dio a conceptos tales como 'langage', 'langue', 'parole', así como a las tareas de la lingüística y a la definición de su objeto de estudio: la lengua, a la dicotomía' sincroníadiacronía, al 'signo' y su 'arbitrariedad', cuando en realidad dicho tratamiento es el producto de una redacción por parte de los editores con base en apuntes de los asistentes a los cursos y a algunas apreciaciones orales por parte del mismo de Saussure. Así las cosas, podríamos decir que el Saussure que dictó los cursos en Ginebra no es el Saussure que se presenta como autor del CLG. De tal forma que la organización (léase distribución) del libro responde más al criterio de los editores que de lo que Saussure hubiera preferido: [...] toutes les parties étant présentées dans un ordre conforme à l'intention de l'auteur (dicho autor no es de Saussure, MM), même lorsque cette intention se devinait plutôt qu'elle n'apparaissait [...] (préface CLG, p. 9). Bally y Sechehaye quisieron soñar un libro así como Borges quiso soñar un hombre, para poder imponerlo a la realidad.

Ahora bien, es conveniente destacar que el trabajo de los editores fue valioso no sólo por la coherencia y la cohesión que supieron darle a las notas de los estudiantes así como a algunas declaraciones sueltas de Saussure ya sea en los cursos o en las cartas personales, sino también por la polémica que suscitaron al asumir la responsabilidad de publicar un libro cuyo pensamiento que lo sustentaba aún estaba en ciernes y cuyos desarrollos sólo podrían ser alcanzados por el propio de Saussure. Esto lo podemos leer en la última parte del prefacio: [...] Nous sentons toute la responsabilité que nous assumons vis-à-vis de la critique, vis-à-vis de l'auteur lui-même, qui n'aurait peut-être pas autorisé la publication de ces pages. Cette responsabilité, nous l'acceptons toute entière, et nous voudrions être seuls à la porter. La critique saura-t-elle distinguer entre le maitre et ses interprètes? (la crítica superficial y apresurada nunca lo hizo, MM). Nous lui saurions gré de porter sur nous les coups dont il serait injuste d'accabler une mémoire qui nous est chère [...]

Perosibien es cierto queel CLGhahechohistoria y de él ha partido un buen número de reflexiones en torno a la lingüística general, se ha hecho caso omiso de la autoría de cada afirmación plasmada

El valor del CLG radica en descubrir e ilustrar el programa saussureano en torno al cultivo de 
la teoría lingüística, es decir, de la lingüística como ciencia. La versión de los editores en la vulgata, en lo que tiene que ver con una teoría de la ciencia, es muy diferente a la de Saussure. Ellos dejan de lado los enunciados que insisten en la oscuridad del objeto de la lingüística, pues deciden no incursionar en la dimensión filosófica que el ginebrino tanto defendió. Las reflexiones sobre lingüística general que bien pueden llamarse filosofía de la lingüística, son la proyección y respectiva elaboración del modelo filosófico de la época, en donde lo filosófico es lo que hoy en día se conoce como lo epistemológico.

Bally y Sechehaye prefirieron plasmar aquellas ideas de Saussure que de cierta forma constituían el 'acervo' de la lingüística. Los editores tan sólo propusieron el esbozo de un programa de búsqueda. Aquellos cuestionamientos 'filosóficos' del ginebrino se quedaron en la sombra. En las notas de cuaderno de los estudiantes así como en algunos escritos de Saussure el cuestionamiento se mantuvo. ¿Por qué no se mantuvo en el curso publicado? Debido a esta polémica y con el ánimo de eliminar los malentendidos de orden conceptual, se ha echado de menos ese libro sobre lingüística general que debería haber escrito de Saussure, para poder emprender de nuevo una interpretación más personal, es decir, de primera mano. Si dicho libro apareciera en este momento (cuyo prefacio podría ser el último párrafo de una carta dirigida a Meillet por el ginebrino en $1894^{2}$ ), provocaría tantas polémicas como el propio Curso y nos obligaría a reconsiderar un camino que un buen número de lingüistas no ha podido ni querido recorrer debido probablemente a las peripecias de su recepción tal y como lo indica Rastier $(2012,8)$ : La réception partielle ou partiale de F. de Saussure par les linguistes ne s'explique pas seulement par le confort que garantissent les vérités académiques: au plan épistémologique, le positivisme ordinaire qui colore les lectures traditionnelles du CLG peine à saisir la dimension interprétative que justifie De l'essence double du langage. Ne serait-ce que parce qu'elles se heurtent aux mêmes incompréhensions, la science saussurienne des textes et la science des textes saussuriens ont ainsi partie liée. L'étude des textes de F. de Saussure par les linguistes gagnerait d'ailleurs à s'inspirer de ses propres études de textes.

\section{2}

Esta sección es la versión española adaptada de algunos apartes de Calvet (1975) y Gadet (1990)

\section{Saussure y la lingüistica general}

Se sabe que en diciembre de 1906 Ferdinand de Saussure dedicó dos horas semanales a dictar un curso sobre lingüística general en la Universidad de Ginebra reemplazando al profesor Joseph Wertheimer. Con esta cátedra complementa su carga académica compuesta inicialmente por los cursos de gramática comparada y de sánscrito. El curso de lingüística general se desarrollará en tres periodos: (i) enero a julio de 1907 (con la asistencia de cinco o seis estudiantes); (ii) noviembre de 1908 a junio de 1909 (con la asistencia de once estudiantes) y (iii) octubre de 1910 a julio de 1911 (con una asistencia de doce estudiantes). ¿Por qué Saussure se ocupa de 'lingüística general'? En el primer capítulo de su obra, Godel traza una semblanza del lugar de la lingüística en la vida de Ferdinand de Saussure (1957, pp. 23-35), concluyendo con un diagnóstico incierto: Redisons-le: aucune obligation extérieure n'a pesé sur lui. S'il n'a fait qu'une année sur deux son Cours de linguistique générale, s'il n'a pas consacré à la théorie du langage tout le temps dont il disposait, c'est en raison de scrupules en partie d'ordre pédagogique, mais aussi, pour une part, tout personnels: «Il me faudrait, dit-il à A. Riedlinger, au moins deux à trois mois de recueillement», et à M. L. Gautier: «Pour aboutir, il me faudrait des mois de méditation exclusive». Plus on s'attache à retrouver les traces de l'activité et des préoccupations de F. de Saussure, plus sa personne s'enveloppe de mystère. El problema es identificar verdaderamente cuál era la relación que se podía establecer entre la reflexión sobre la lingüística general y los demás trabajos que él

2 Cela finira malgré moi par un livre où, sans enthousiasme ni passion, j'expliquerai pourquoi il n'y a pas un seul terme employé en linguistique auquel j'accorde un sens quelconque. Et ce n'est qu'après cela, je l'avoue, que je pourrai reprendre mon travail au point où je l'avais laissé. 
realizaba como por ejemplo los Anagramas [ver a este respecto los comentarios de J. Starobinski (1971)]. Las confidencias de Saussure en sus cartas, por ejemplo a Meillet, muestran a un Saussure ajeno al Curso quien parece no interesarse en la lengua en general, y confiesa que la necesidad de reformar el vocabulario lingüístico menoscaba su placer histórico. Numerosos hechos confirman este perfil. Sabemos que Saussure era un hombre de lenguas más que de la lengua. A los 16 años maneja ya el francés, el inglés, el alemán, el griego y el latín y estudia el sánscrito. En noviembre de 1891, en la primera conferencia en la Universidad de Ginebra, Saussure ataca a estudiosos como Friedrich Müller, que se ocupan de la lengua en general: Ce ne sont pas des linguistes comme Friedrich Müller, de l'Université de Vienne qui embrassent à peu près tous les idiomes du globe, qui ont jamais fait faire un pas à la connaissance du langage; mais les noms qu'on aurait à citer dans ce sens seraient des nom des romanistes comme M. Gaston Paris, M. Paul Meyer, $M$. Schuchardt, des nom des germanistes comme $M$. Hermann Paul, des noms de l'école russe s'occupant spécialement du russe et du slave, comme M. M.N. Baudoin de Courtenay, M. Kruszewski [ELG, p. 147].

No obstante esta preferencia por la descripción concreta, por la investigación histórica, la encontramos en el momento en que dicta sus tres cursos de lingüística general y en sus trabajos sobre los anagramas. Sin embargo esto no aclara su personalidad. Hay una suerte de paradoja en la producción de los años 1905-1910. Por un lado, dicta un curso casi improvisado, con pocas notas y sin ninguna redacción. Por otro lado, redacta con dedicación varios cuadernos dedicados a los anagramas cuadernos prácticamente listos para su publicación. Entonces, (i) nunca habla de las investigaciones sobre los anagramas en sus cursos. De hecho, la palabra 'anagrama' no figura en el CLG; (ii) prácticamente hay que esperar los años 60 para oír hablar por primera vez de dichas investigaciones y (iii) es el trabajo sobre la lingüística general el que le dará renombre y reputación póstumos a Saussure. Desde las conferencias inaugurales de 1891, se observa un interés por algunos puntos fundamentales: el aspecto social de la lengua, la oposición entre lengua/habla, sincronía/diacronía, etc. Sus ideas son fijas pero con frecuencia chocan con el problema terminológico. Así las cosas, en el cuarto fascículo de la edición crítica de Engler, notas tituladas sur les difficultés de la terminologie (3300, 3301); así como notes pour un livre sur la linguistique générale $(3295,3296)^{3}$, este libro del cual le habla a Meillet.

Teniendo en cuenta lo anterior, podemos proponer una hipótesis. A través de sus diferentes trabajos, a través de la carta de 1894 dirigida a Meillet, a través delas investigaciones apasionadas sobre los Anagramas e incluso los Nibelungen, se adivina a un Saussure cuyos intereses se alejan evidentemente de la fundación de una lingüística general pero que tiene necesidad, para finalizar los trabajos que ha emprendido, de un terreno conceptual sólido. La lingüística general constituía en el fondo, para él, un instrumento necesario y los cursos representaban la búsqueda de este instrumento, esto es, eran un medio y no un fin en sí mismos: posiblemente inventamos una lingüística general autónoma a partir de Saussure, mientras que para él, solo era un pasaje obligado hacia otra cosa. Una vez puestas las bases de una lingüística general satisfactoria, Saussure hubiera podido concentrarse en sus trabajos históricos que constituían su verdadera pasión: es al menos la lección que se saca de los manuscritos. Un espejismo colocó al CLG en el primer plano de los trabajos saussureanos. Otro espejismo lo transformó en el manifiesto de la lingüística general, en fundamento teórico de una ciencia autónoma del lenguaje. ¿Saussure vs Saussure?, ¿y si la constitución y devenir del CLG estuviera en contravía con las ideas del hombre Saussure? Y ¿qué sucedería si entre la imagen Saussure y el hombre Saussure solo existiera una proyección ideológica además de las imperfecciones epistemológicas de dos generaciones de lingüistas? Rastier (2016, 7) aporta una respuesta: Actualmente Saussure es, con Chomsky, el único lingüista que sobresale del círculo disciplinario para representar (a veces vagamente) su disciplina a la vista de los otros. Pero incluso entre los

3 Estos textos se reproducen en los Escritos de lingüística general, (2004, versión española): Tercera parte, ‘Otros escritos de lingüística general', item II, Antiguos documentos números 10a- 10b- 10c- 12a- 12b. 
lingüistas Saussure sigue siendo mal comprendido y hasta incomprendido [...] La recepción parcial o restringida de Saussure por los lingüistas no se explica solamente por la molicie que garantiza las verdades académicas. En el plano epistemológico, se le hace difícil al positivismo ordinario que colorea las lecturas tradicionales del Curso de lingüística general (en adelante CLG) comprender la dimensión interpretativa que justifica De la esencia doble del lenguaje [De l'essence double du langage]. La ciencia saussureana de los textos y la ciencia de los textos saussureanos se enfrentan a las mismas incomprensiones, aunque sólo sea porque sufren un impedimento similar. El estudio de los textos de Saussure por los lingüistas ganaría, además, si se inspirase en los estudios del propio Saussure sobre los textos.

Tal parecequeel proyectodellingüistaginebrino era el de forjar un instrumento analítico para trabajar en varios dominios. Al tiempo, planteó las bases de una cierta lingüística moderna y su genio se valida en este azar: buscando las Indias, Colón encontró a América.

\section{Cómo se hizo el CLG}

Imaginemos por un momento a un Saussure que no hubiera sufrido de 'grafofobia' y que hubiera escrito un libro a partir de su reflexión sobre la lingüística general. Se podría pensar que dicho libro sería semejante a sus otros escritos especializados y que nunca hubiera alcanzado el impacto que tuvo el CLG. Así las cosas, valdría la pena saber cómo se escribió el CLG.

En el prefacio, Bally y Sechehaye presentan sus razones. Ante todo, les tocó hacer un trabajo crítico sobre las notas de los estudiantes, comparar punto por punto y para cada uno de los tres cursos las versiones en ocasiones divergentes. Conscientes de la dificultad de transponer por escrito un texto oral, ellos renuncian a publicar todo. Rechazan igualmente la opción que consistía en editar solo uno de los cursos, ya que el programa seguido no era el mismo de un año a otro, y se hubieran perdido elementos interesantes; o editar solo fragmentos escogidos, lo que hubiera ocultado todo desarrollo seguido. Los editores adoptan una decisión más atrevida, "intentar una reconstrucción, una síntesis, a base del curso tercero, utilizando todos los materiales de que disponemos" siendo conscientes de los límites de su empresa. La forma definitiva que le dan a su obra de 317 páginas presenta cinco partes: una introducción en donde se exponen los problemas correspondientes al objeto de la lingüística, a la cual se le añade un apéndice de fonología; una primera parte de 'Principios generales' en donde son tratados el signo y la sincronía; una segunda parte, 'lingüística sincrónica' de donde provienen todas las nociones relacionadas con la determinación de la unidad, la identidad, el sistema, el valor, las relaciones y el mecanismo; una tercera parte, 'lingüística diacrónica' en donde se tratan los cambios la analogía y la etimología popular; vienen enseguida la cuarta y quinta partes, 'lingüística geográfica' $\mathrm{y}$ 'cuestiones de lingüística retrospectiva $\mathrm{y}$ conclusión respectivamente. ¿En qué consisten exactamente las fuentes que tenían a la mano los editores para llevar a cabo este trabajo? Godel, en su libro ${ }^{4}$ describe tres tipos de fuentes: (i) Notas del propio Saussure, una buena parte de ellas había sido redactada hacia 1894 año en el cual él estaba muy interesado en cuestiones de lingüística general (cf. Carta a Meillet) y probablemente esbozó una obra. De 1894 son también las 70 páginas de notas destinadas a un homenaje al lingüista americano William D. Whitney. Saussure quiso aprovechar esta ocasión para presentar sus ideas pero al final no fueron enviadas. De todas estas notas redactadas a lo largo de los años, una parte se perdió e incluso se destruyó; (ii) Notas destinadas a la preparación de los tres cursos, no muy numerosas, breves y solo corresponden a algunas lecciones; (iii) Notas tomadas por los estudiantes a lo largo de los tres cursos que difieren a la vez en la materia, el plan y la forma. Los editores intentaron reunir todos los cuadernos disponibles, lo que era posible dado que en el tercer curso por ejemplo, la asistencia no superaba las cuatro o cinco personas. 
Los profesores Godel y Engler ${ }^{5}$ se ocuparon con profundidad de las fuentes. El primero, después de trazar un panorama de los intereses de Saussure por la lingüística general, describe todos los materiales a partir de los cuales se diseñó el CLG, elaborando una amplia reflexión sobre los principales problemas de interpretación. Engler por su parte, presenta un texto distribuido en seis columnas (cuadro 1 Es el scan Curso Engler). La columna izquierda [1] contiene el texto del CLG, dividido en cortos fragmentos numerados de 1 a 3281; las columnas [2], [3] y [4] presentan las fuentes conocidas por los editores, colocando frente a cada fragmento su fuente identificable en los cuadernos de los estudiantes (con un sistema de remisiones que le permitiría a quien quisiera, seguir la secuencia de la fuente); las columnas [5] y [6] presentan textos desconocidos en 1916 y hallados por Godel.

Gracias al trabajo de Engler, podemos trazar la historia de este texto: de la exposición oral de Saussure a las notas que toman los estudiantes, de la lectura de las notas hechas por los editores en la redacción del CLG. Queda pendiente evaluar los efectos de estas transmisiones sucesivas en el texto definitivo.

El plan fue establecido sobre la base del tercer curso, aunque el orden no haya sido conservado en términos generales. Podemos en efecto resumir el plan seguido por Saussure en este tercer curso de la siguiente forma: de la diversidad de las lenguas a la lengua, de la lengua a lo lingüístico. Los editores decidieron comenzar por la lengua y de remitir las lenguas $y$ su diversidad al final de la obra (partes 3, 4 y 5).

El tercer curso es la fuente esencial de la introducción, de la primera, segunda y cuarta partes. El primer curso es la fuente de la tercera parte y de los apéndices. El segundo curso fue utilizado como apoyo y es la fuente de algunos capítulos. En cuanto a las notas manuscritas de Saussure, los editores no hicieron más que los elementos al interior del texto establecido independientemente. Las notas de los estudiantes fueron retocadas de manera tal que pudieran integrarse en el texto elaborado, que presentan, la mayor parte del tiempo, una paráfrasis muy próxima. Buena parte de las modificaciones aportadas es mínima así como los ajustes terminológicos ya que varios de los términos solo aparecen a partir del Curso III. Los editores explicitaron también pasajes oscuros o demasiado alusivos, escasos a decir verdad, adjuntado o sustituyendo ejemplos, añadiendo comentarios a los esquemas. Solo algunas modificaciones aportadas ponen en duda la fidelidad a las fuentes, de una manera más o menos grave que va de un ligero endurecimiento del pensamiento a una modificación del orden de presentación de los conceptos o a una reducción de la importancia de una parte en relación con lo que se espera de las fuentes.

Para ilustrar lo que se ha venido diciendo, se citará el ejemplo del enunciado conclusivo, uno de los más citados del CLG: «La linguistique a pour unique et véritable objet la langue envisagée en ellemême et pour elle-même» (p.317). Dicho enunciado fue totalmente fabricado por los editores. Aunque no sea contrario al espíritu del Curso, acentúa el exclusivismo y no causa sorpresa que en su nombre varios lingüistas estructuralistas hayan interpretado el pensamiento saussureano excluyendo todo dinamismo del sistema, todo condicionamiento social, todo lazo con la historia.

No obstante, solo se le puede rendir homenaje apoyado en el trabajo hecho por los editores que supieron, la mayoría del tiempo, dejar de lado sus propias opiniones para penetrar en el pensamiento de Saussure. En cuanto a ellos, no tienen duda de su fidelidad, como lo testimonia el hecho que solo modificaron puntos de detalle en la segunda edición, en 1922.

La cuestión del orden de presentación está presente en la propia génesis del pensamiento saussureano. Si este punto constituyó un

5 Edition critique du Cours de linguistique générale de Ferdinand de Saussure, Wiesbaden, Otto Harrassowitz, 19671974. 
problema para Saussure, luego para los editores, no haya nada sorprendente en el orden de la presentación haya constituido un problema para nosotros, en nuestro propio trabajo: ¿qué orden adoptar? Veamos las posibilidades que están a mano: (i) El orden del CLG que dependería de una interpretación previa e inevitablemente en dicha interpretación hay equívocos. Como el orden del Curso no corresponde al orden natural y real de los cursos y menos como se cree, al del tercero, no hay ninguna razón válida para seguir este orden ya que no permite una comprensión global pues no se presentan los lazos teóricos entre los conceptos; (ii) El orden de las Fuentes que obligaría a escoger entre cualquiera de los tres cursos y llevaría a la larga a desconocer lo que no se incluye en alguno de los cursos; (iii) El orden 'reconstruido' de la génesis de los conceptos en la reflexión saussureana.

Otra posibilidad consistiría en proponer un orden lógico, teniendo en cuenta las posiciones de cada concepto en la configuración teórica de conjunto. No obstante, esto plantearía dos problemas: (i) en primer lugar el de la interpretación, ya que esta depende del punto de vista que se escoja y (ii) la más difícil: o es posible desarrollar el CLG de manera lineal, de tomar una noción y de deducir todo de ella. Por otra parte tampoco se puede llegar a un punto de vista único: enfoques diversos, no contradictorios, contribuirían a establecer una noción surgida de varias problemáticas a la vez. Así las cosas, ¿cómo podemos en la actualidad evaluar el interés de Saussure? Esto nos lleva a formular una pregunta esencial y complementaria: ¿qué se espera de una teoría lingüística? Para responderla habría que focalizar tres objetivos. El primero, explicar la mayor parte de hechos de lengua; el segundo, comprender lo que en el funcionamiento del lenguaje, permite que se puedan crear enunciados nuevos, así como la palabra cotidiana que en los usos particulares, obedece al sistema constitutivo de cada lengua y finalmente, el tercero sería definir la especificidad del trabajo del lingüista entre las diferentes disciplinas que se ocupan del lenguaje.

\section{3}

Para la primera parte de esta sección se toman apartes de dos textos del profesor José Polo titulados "Presencia de Saussure en el mundo hispánico" y "Traducciones al español del CLG de Saussure"; se modifica el orden del contenido de los artículos para intereses de la presentación, se tiene en cuenta la variable cronológica y los respectivos representantes. La información de la profesora Nethol y del caso latinoamericano fue preparada por el editor.

Cuando se habla de la trascendencia de la obra científica de este genial lingüista, considerado creador de la Lingüística Moderna, suele estudiarse al mismo tiempo la enorme influencia de su pensamiento por doquier. En los repasos geográfico-históricos saussurianos a que estamos acostumbrados, desfilan lustrosos algunos países, o algunos ámbitos culturales, presentados como adelantados en la investigación historiográfica de Saussure. Pues bien, uno de los que no aparece en primera línea en este respecto es España (o, en general, el mundo hispanohablante).

\section{Juan Manuel Dihigo (1866-1952)}

Fue el primer estudioso, cubano, que en el ámbito de la lengua española escribió sobre el Curso de Saussure. En la Revista de la Facultad de Letras y Ciencias de la Universidad de la Habana (vol. XVII-3/1913, pp. 190-193) dentro de la sección Necrología, publica una hermosa nota "Fernando de Saussure". Con ocasión de una reforma en los planes de estudios de letras en su universidad, le escribe Dihigo a Saussure y este le contesta en carta con fecha del 16 de noviembre de 1906 y le recomienda que deje para el final de la carrera las generalidades, que en los primeros cursos interesa el contacto con los hechos concretos. En el volumen XXIV-1/1917 de la revista citada, pp. 96-97, publica Dihigo su reseña al flamante Cours de linguistique générale (1916). Escrito más apagado que el anterior, menos vibrante; pero persiste su admiración por el maestro ginebrino y se

6 Cuadernos de Investigación Filológica CIF, tomo XVIII, fasc. 1 y 2 (1992), pp. 183-196. 
fija en algunos conceptos: sincronía/diacronía, etc.

\section{Dámaso Alonso}

En el archivo de este autor, se encuentra un cuaderno que contiene la traducción resumida de la obra por antonomasia de Saussure. Unas veces se trata de la mera síntesis del texto francés y otras de traducción completa de determinados pasajes; comprende desde el comienzo del libro hasta el final de la primera parte (o sea, hasta la página 140 de la edición original, 1931, que corresponde a la 174 en la versión de Amado Alonso); así que lo "traducido" por Dámaso Alonso representa más de un tercio de la obra completa.

\section{Amado Alonso}

En 1945 publica nuestro autor, en la Editorial Losada, su traducción del Cours de Saussure: Curso de lingüística general. Además de la versión, Amado Alonso anota la obra y le pone un espléndido "prólogo a la edición española", pp.7-30 [El Prólogo hace parte del presente dossier, MM], simultáneo prácticamente con su artículo "La doctrina lingüística de Ferdinand de Saussure", en La Nación de Buenos Aires (12 de agosto de 1945).

\section{Cuadro cronológico resumen}

1916: fecha de publicación del Cours;

1917: en el tomo IV de Revista de Filología Española, pág. 85, ficha 5024, aparece la obra de Saussure;

1921: La Paraula, de Ivón l’Escop;

1922: de nuevo RFE: tomo IX, pág. 202, ficha 11403;

Recueil des publications scientifiques de Ferdinand de Saussure (c. Winter, Heidelberg, 1921);

1933: Pompeu Fabra presta atención, aunque no tan declarada y sistemática, a Saussure;
1945: Amado Alonso traduce, anota para lectores hispanohablantes y prologa la obra clásica de Saussure: Cours de linguistique générale (1916, 1922, 1931).

Hasta aquí Polo. Con respecto a la recepción, divulgación y estudio del pensamiento saussureano en otros países de habla hispana, son escasas las referencias debido, en parte, a las razones esgrimidas por De Lemos et al. (2003, 173): On peut même affirmer qu'en Amérique Latine, Saussure n'est pas allé au-delà des salles de classe, lieu où il est mentionné comme 'celui qui a proposé de célèbres antinomies' qui se sont perpétués comme 'les nouveautés' qu'il a ajoutées l'histoire de la pensée linguistique [...] En tant que 'nouveautés', les dichotomies entrent dans 'l'ordre du jour' - un modisme dont le destin est prévisible: les nouveautés sont révolues, même si elles restent comme 'quelque chose en plus' dans l'histoire de la réflexion sur le langage. Dans ces noyaux de formation se perpétue une lecture médiatisé par un sous-texte languissant et paralysant de la pensée du 'père de la linguistique scientifique'[...] La réception des manuscrits, l'existence d'importantes exégèses et d'apparats critiques ne sont pas inconnus en Amérique Latine la question est qu'ils sont écartés comme appartenant au passé. Ce manque d'intérêt pour le retour, la pensée de Saussure semble être le reflet de la parution de la Grammaire Générative, la fin des années 1960, mais aussi et surtout de ce qui n'a pu être lu dans le Cours, i.e., la nature révolutionnaire des idées et des concepts introduits par Saussure ${ }^{7}$.

Estanislao Sofia $(2016,1-2)$ en la presentación del dossier que le dedicó la revista Signo y Seña a los cien años del CLG, sigue preguntándose por los modos de recepción que Saussure ha tenido en América Latina. Se destaca la labor divulgativa y analítica de tres estudiosos, Amado Alonso (desde la Argentina), Eugenio Coseriu (desde el Uruguay) y Joaquim Mattoso Câmara (en el Brasil) en la apropiación del pensamiento saussureano en nuestro contexto tropical. Sofia plantea tres preguntas que aún no han tenido una respuesta satisfactoria y

7 A propósito del centenario del CLG, hemos hecho las consultas a colegas lingüistas de los países hispanos de América del Sur y de Centroamérica (incluyendo a México), preguntando si en sus países había individuos o grupos de investigación dedicados a estudiar la obra de Saussure. La respuesta fue negativa. 
cuyas posibles respuestas pueden trazar nuevos horizontes investigativos: ¿Tuvieron Alonso, Coseriu y Mattoso Câmara un rol sobresaliente en todos los países? ¿Qué matices del pensamiento saussureano rescataron unos y otros? ¿Se influyeron mutuamente?

Comentaremos a continuación el valioso aporte de la profesora argentina Ana María Nethol con su libro Ferdinand de Saussure. Fuentes manuscritas y estudios críticos (1977). Una primera edición del libro había aparecido en el año de 1971 y su contenido presentaba solo estudios críticos sobre la obra del ginebrino entre los que se encontraba el texto "La naturaleza del signo lingüístico" de Emile Benveniste. En la nueva edición, la de 1977, se le añadió una unidad más [primera parte, pp. 13-101] que consta de los manuscritos de los alumnos de Saussure: los apuntes de la Introducción pertenecen al curso de lingüística dictado en la Universidad de Ginebra entre 1908 y 1909, compilados y ordenados por Robert Godel y publicados en 1957. La segunda parte del libro [pp. 103-253] está compuesta por los siguientes estudios críticos: Proceso de producción de una teoría lingüística y semiológica (Ana María Nethol, incluida en este dossier); Actualidad del saussurismo (A.J. Greimas); La teoría del lenguaje de Ferdinand de Saussure (Robert Godel); El sistema lingüístico de Ferdinand de Saussure (Rulon S. Wells); Saussure, medio siglo después (Emile Benveniste); Lengua y habla (Louis Hjelmslev); Los anagramas de Ferdinand de Saussure (Jean Starobinski) y una sección de documentos para la historia de algunas nociones saussureanas (pp. 249-253) que contiene, entre otras cosas, la reseña que Leonard Bloomfield hizo del CLG. No se incluyó el texto de Benveniste "la Naturaleza del signo lingüístico" por considerarlo parte de una polémica ampliamente discutida que se centra en un pasaje del Curso de lingüística general y cuya interpretación es tan dudosa como la intención de Saussure de volcar ciertas afirmaciones sobre el signo.

En la 'Advertencia', la profesora Nethol ilustra los propósitos discursivos de la publicación que se transcriben a continuación.

Por último (y esto constituye casi una segunda advertencia), algunas palabras sobre el sentido y los motivos de esta publicaci6n; cuando en 1971 armé la compilación a que hago previamente referencia, la perspectiva desde la que-la organicé era distinta a la actual. Por entonces pienso que existía la tendencia a creer en una especie de verdad o realidad especulativa, cuya producción se encerraba en el vicioso circuito de intercambio que los textos iban armando entre sí. Unos cuantos núcleos librescos constituían las fuentes de salida a las que se adherían nuevos textos cuyo campo referencial era siempre la escritura y los datos de la "otra realidad" el pretexto para remitir $a \sim$ sos nódulos. Por entonces y aun antes, un grupo de trabajadores en las ciencias sociales argentinos y latinoamericanos comenzaron a orientar su práctica hacia una perspectiva transformadora, canalizando los problemas fundamentales y descartando las discusiones estériles para encarrilarlas a un proyecto científico liberador: se señalaron objetivos a los que tenía que abocarse todo científico consciente en el marco de ese proceso. Para enfrentar al imperialismo había que ir creando condiciones de existencia y proyectos concretos de una ciencia latinoamericana $y$ argentina $y$, en el plano interno, encontrar los objetivos específicos dentro de cada zona de trabajo.

Durante este lapso cobró vigor en la Argentina un proyecto político que, junto a instancias concretas de trabajo, intenta marchar hacia un mismo objetivo. Dentro de estas perspectivas muchos de nosotros fuimos cambiando, por una parte, el punto de vista que nos situaba frente a las producciones provenientes en su mayoría de países centrales. y, por otra; abriendo un amplio abanico de interrogantes sobre el papel de la ciencia en un país dependiente y proyectos para implementar actividades acordes con las necesidades del proceso.

Sibien no es este el espacio adecuado para desarrollar la gama de interrogantes que nos formulamos, creo que puede ser ilustrativo señalar algunos de ellos. En primer lugar, la significación y alcances políticos del campo científico que abordamos, la definición y puesta en acción de una ciencia transformadora y las prioridades para llevar adelante esa práctica. En lo que atañe a los textos y materiales provenientes de centros de poder, su revisión, crítica y reubicación en función de las condiciones científicas y políticas de producción de las que eran emergentes y su posible utilización en función de dichas tareas prioritarias. 
En la Argentina, la situación de la ciencia linguistica reproduce las condiciones en que se encuentran las ciencias sociales en general: durante las décadas de 1950 a 1970 nuestros estudiosos $e$ investigadores retoman las mismas temáticas y adoptan los sucesivos métodos y temas que provienen de centros cuyas prioridades científicas y desarrollo tecnológico poco tienen que ver con los nuestros. La actividad científica se encuentra en un grado tal de dependencia que la producción de trabajos disminuye con respecto a años anteriores y se encierra en el círculo vicioso de la discusión entre tendencias (que no son otras que las importadas) y, a lo sumo" en la realización de cursillos donde los más "adelantados" - o los más pacientes-reproducen lo que han aprendido.

Dentro del mismo movimiento que comienza a cuestionar el papel de las ciencias sociales y sus procedimientos y temáticas vigentes, un grupo de lingüistas argentinos y deotros países latinoamericanos se plantean la práctica de esta ciencia como una imperiosa necesidad política. Basta una ojeada de conjunto sobre los problemas más generales: a la incidencia de las políticas del lenguaje en el ámbito sociocultural, a las consecuencias nefastas de la represión sobre el lenguaje, a la hegemonía política de un dialecto sobre otro, a la influencia del lenguaje de los medios de comunicación, a la carencia de planificación adecuada para la enseñanza del lenguaje en la escuela primaria y media (para sólo citar ejemplos sobresalientes) para ver cuáles son los canales de trabajo que debemos seguir para poder abrir brechas coherentes que, apartándose de polémicas estériles, construyan objetivos y prácticas sólidas.

A partir de estos planteos, reeditar un libro dedicado a Ferdinand de Saussure adquiere un nuevo sentido: un sentido instrumental, donde la lectura del clásico y las interpretaciones de su obra no constituyen la posibilidad cerrada de conocer a fondo una teoría para legitimarla o detractarla; constituye una perspectiva abierta para extraer, profundizar, explicar y aplicar los elementos que sirven de ella en función de las necesidades que nos imponen la observación empírica y la acción sobre la realidad a partir de la posición histórico-política que asumimos.

Para cerrar este acápite, vale la pena mencionar el caso colombiano. Con excepción de la profesora Claudia Mejía (cuya formación, estadía y trabajo en Suiza la puso en el centro de los estudios saussureanos y se considera un caso aparte) ${ }^{8}$, ningún estudioso colombiano se ha concentrado en la obra de Saussure. Los especialistas en diferentes variantes teóricas como es el caso de José Joaquín Montes, Carlos Patiño Rosselli, Lucía Tobón de Castro, Felipe Pardo, Jorge Páramo P., Luis Ángel Baena, por citar solo los más reconocidos en el ámbito nacional, se ocuparon, como la mayoría de lingüistas, en parafrasear el CLG, en comentarlo en su ejercicio de la docencia y en algunos casos llegaron a disertar sobre temas puntuales como el signo lingüístico, el objeto de estudio de la lingüística o la particularidad de las dicotomías saussureanas. Nada más. Hay incluso toda una serie de trabajos eruditos, aislados y sin continuidad que aportan datos, valiosos por supuesto, pero carecen de todo sustento explicativo y de proyección ${ }^{9}$. Sofía (2012) destaca el aporte de Héctor Peña titulado "Trabajo de la lengua/Trabajo de sentido: la lección de Alcira Saavedra sobre Ferdinand de Saussure", indicando que dicho trabajo "explora las alternativas de la transmisión concreta de las ideas de Saussure en Colombia tomando por ejemplo el caso de la profesora Alcira Saavedra, de cuyos cursos en la Universidad de los Andes de Bogotá Peña fue asiduo asistente. En un doble homenaje que celebra a un tiempo la publicación del Curso y las lecciones de Saavedra, el autor presenta una lectura singular (la de Saavedra) de ideas que habrían podido considerarse clásicas e indiscutibles: los conceptos

8 Cf. «Survivre dans les mots». Extrait de la Postface au livre Parentalité stérile et procréation médicalement assistée. Le dégel du devenir, de Claudia Mejía Quijano, François Ansermet et Marc Germond. Eres, 2006.

9 Citamos algunos de ellos: Francisco Zuluaga Gómez (1986). El Curso de Lingüística General: Reflexiones sobre el libro y el autor. Lingüística y Literatura 10, pp.63-81; Doris Santos (2005). Un análisis paradigmático de los aportes de F. de Saussure y N. Chomsky al campo de los estudios del lenguaje. Forma y Función 18, pp. 215228; César Romero Farfán (2014). Lección de Lingüística. Consideraciones en torno de Ferdinand de Saussure: Asidero de la lingüística actual. Cuadernos de Lingüística Hispánica, 24, pp. 13-24. 
"lengua", "saber" y "sentido", en particular, se verán redefinidos en una articulación original que Peña reproduce con claridad". Vale la pena hacer un seguimiento de dicho aporte ${ }^{10}$.

\section{4}

El dossier sobre Saussure y el Curso de lingüística general está compuesto por cuatros secciones internas. La primera sección es la de 'textos clásicos' que contiene tres trabajos dirigidos al entorno hispano. Se trata del 'Prólogo' que el profesor Amado Alonso escribe para la traducción española del CLG (1945), texto al cual no le haremos comentario pues es ampliamente conocido y de él ya se han ocupado ilustres estudiosos. El artículo de la profesora Ana María Nethol dedicado a la teoría semiológica de Saussure y titulado 'Proceso de producción de una teoría lingüística y semiológica', es un intento por mostrar que en Saussure existe un arduo trabajo teórico cuya base es la apoyatura semiológica y donde el estudio de la lengua ocupa un lugar más, si bien privilegiado, por lo avances teóricos (evidentemente no casuales) dentro de la amplia gama de sistemas de signos. Esta contribución hace parte de un trabajo mayor, ya lo mencionamos en la sección precedente, un libro dedicado a divulgar el pensamiento saussureano y a poder consolidar en Latinoamérica un movimiento crítico que no se limitara al sentido instrumental y erudito de conocer y entender dicho pensamiento, sino además ser capaces de ponerlo en perspectiva.
El tercer trabajo, a manera de primicia, es la Introducción del libro que aparecerá el próximo año en las prensas dela Universidad de Antioquía a cargo de la profesora Claudia Mejía, única especialista de Saussure en Colombia, titulado Lingüística general de Ferdinand de Saussure. El primer curso. Introducción. Se destaca que hasta hoy, ningún curso completo de Saussure había sido traducido al español. El lector no sólo encontrará aquí la primera traducción al español del curso de lingüística general de 1907, sino también la primera edición integral en francés de los únicos manuscritos de primera mano que se conservaron, y que seguían todavía inéditos: uno porque apenas se descubrió en el 2010, el otro porque estaba escrito en taquigrafía y sólo unas cuantas páginas habían sido descifradas y publicadas por Rudolf Engler desde 1968. Esta edición tiene pues el privilegio de llenar dos vacíos en ambas lenguas, gracias a un valioso trabajo de equipo.

La segunda sección, 'artículos', consta de nueve trabajos. El primero se titula Le Cours de linguistique générale et l'histoire du saussurisme (AnneGaëlle Toutain). Después de presentar algunas particularidades discursivas con respecto a las posturas suscitadas por la autoridad y fama del Curso de lingüística general, las posturas de sus seguidores y detractores, la confrontación entre el texto del Curso y el texto de los manuscritos, los autógrafos, la profesora Toutain muestra que el CLG es el resultado de una lectura de Saussure y se inscribe como tal en una historia

10 Dice así el resumen del artículo: El centenario de la publicación del Curso de lingüística general encarna la complejidad de un problema grave: la de los que definen o ponen en evidencia la alteración de un estado de cosas. Hoy, a diferencia de cualquier otra conmemoración previa al descubrimiento de la Orangerie de finales del siglo pasado, debemos no perder de vista la importancia de lo que otro Saussure, no uno sólo diferente en virtud de intereses disímiles o de formulaciones divergentes en relación con el textus receptus del Curso, nos obliga a saber de su lección sobre "lingüística general". Este otro Saussure, al que no sería desmesurado presentar como un "Saussure otro", aparecía con especial fecundidad en las lecciones de una profesora colombiana, Alcira Saavedra Becerra, quien lo situaba como fundamento de una exploración radical: la del trabajo del sentido, la de aquello que habría que mostrarle al pensamiento como trabajo propio, aun en contra de sus propias resistencias, denegaciones, recuperaciones o forclusiones. El pensamiento debería saber que lo que sabe es un saber de lengua y que este saber, incluso sin saberse (incluso evitándolo como saber o incluso sin formularlo como saber de algo), es condición de cualquiera de sus posibles. A partir de una "lectio saussureana" que aparece, con todo y los avances filológicos que sustentan sus estudios en la actualidad, irreductible a la pureza de una doctrina (i.e. ajena a la estabilidad de lo dicho por el maestro), este trabajo busca exponer otra lección: aquella en la que vieron la luz, en Colombia, algunos otros "saussures" —el del trabajo del sentido, en primer lugar-, gracias a la enseñanza de Alcira Saavedra B. [2016, 37] 
que este inaugura y además supera y cuya característica principal es lo que se denomina en psicoanálisis principio de repetición. Para tal efecto se apoya en dos de las cuatro "recepciones de Saussure" establecidas en Puech (2005), a saber, (i) las reseñas, (ii) los Círculos, (iii) el estructuralismo generalizado y (iv) los trabajos filológicos inaugurados por los estudios de Godel (1957) y de Engler (1967/1974), reemplazando el análisis de la primera por el estudio de cómo se constituyó la redacción del CLG y dejando de lado la tercera para concentrarse propiamente en el campo lingüístico.

El segundo artículo, Un bon exercice de lecture $d u$ Cours de linguistique générale (Claire Forel), describe cómo, en el marco de un seminario titulado 'Reading Saussure and Chomsky', se suscitan problemas de interpretación no solo al transmitir el pensamiento saussureano sino también al confrontarlo con el pensamiento chomskyano. Se parte del supuesto además de que Chomsky solo pudo leer el CLG y se toma este último como texto de referencia que será confrontado a las notas de los estudiantes, a las del mismo Saussure así como a la Collation Sechehaye ( $c f$. Sofia, 2015). Para tal efecto, se escogen tres pasajes del Curso: (i) las primeras líneas; (ii) el famosos circuito del habla (ambos pasajes hacen parte de la 'Introducción') y (iii) la célebre comparación de la lengua con el juego de ajedrez (CLG, pp. 125 y 153) que llamó en particular la atención de Chomsky. Se ven ejemplos de adjunción o supresión en relación con las notas de los estudiantes, reformulaciones que en cierta forma mejoran la lectura del pensamiento saussureano para comparar al tiempo la manera como Chomsky lee e interpreta la obra de Saussure en especial en su Knowledge of language (1986).

El tercer artículo, Les cours de linguistique générale de Ferdinand de Saussure, un projet de Paul-F. Regard et Antoine Meillet (Claudia Mejía) presenta un proyecto paralelo a la edición del CLG que en ese momento no fue llevado adelante por sus diseñadores y permaneció desconocido, pero más tarde fue desarrollado espontáneamente por varios lingüistas a lo largo del siglo pasado. Se trata de la concepción de este proyecto por dos excelentes seguidores de Saussure, a través de cartas no publicadas recientemente encontrados en el Castillo de Vufflens (Mejía Quijano, 2015). Estas cartas se complementan con otras cartas ya publicadas, lográndose captar con mayor claridad las cuestiones teóricas de este proyecto paralelo, que sigue siendo relevante.

El cuarto artículo, El Curso de lingüística general de Saussure y su importancia en el desarrollo de la lingüística estructural (Gerda Haßler) es una aproximación histórica en donde se destacan varios elementos. En primer lugar, la influencia que tuvo el CLG en la consolidación del pensamiento estructuralista (i.e. la lingüística estructural) convirtiéndose además en el texto de 'referencia' del estructuralismo. En segundo lugar, destaca que existen representantes de la serie llamada pre-estructural cuyos textos que, siendo estructurales, no se consideran como fundadores del estructuralismo e insiste en que es importante no dejarse guiar por una idea teleológica, sino apoyarse en textos concretos con finalidad histórica. Finalmente, a pesar de la afirmación que el Curso ha desnaturalizado el proyecto auténtico de Saussure, se confirma que el concepto del valor lingüístico está presente en todas las versiones de su doctrina: en sus trabajos sobre la lingüística histórico-comparativa, en el Cours de linguistique générale editado por Bally y Sechehaye y en los manuscritos auténticos. Con la introducción de una naturaleza formal de la lengua, Saussure refuncionalizó un enfoque existente en teorías anteriores y lo ha puso en el centro de su doctrina. El presente artículo es la reescritura y actualización que hizo la autora de un artículo previo queriendo hacer un aporte notable a nuestro número.

Ontologie et épistémologie de la linguistique dans les textes originaux de Ferdinand de Saussure se titula el quinto artículo, escrito por el profesor Simon Bouquet. En dicho texto, el autor enfatiza en que la lectura que presenta de los textos de Saussure es hecha en calidad específica de lingüista, focalizando la utilidad que el pensamiento saussureano le aportaría en realidad a la ciencia lingüística. Se trata de un ejercicio claramente epistemológico que pretende, 
apoyándose en los nuevos escritos de Saussure, describir los rasgos esenciales del verdadero programa epistemológico saussureano para lo cual adopta dos puntos de vista: (i) hacer una lectura lo más objetiva posible del corpus original con el fin de definir los principios generales del programa saussureano y (ii) presentar una lectura más subjetiva buscando proyectar dicho programa en la perspectiva de lo que pareciera, fuera su posible evolución teniendo en cuenta el estado actual de la lingüística.

El siguiente artículo Some remarks on Chomsky's reading of Saussure (Frederick J. Newmeyer) se ocupa de la posible interpretación que Chomsky pudo haber hecho del concepto saussureano de lengua cuando afirma en su libro de 1986, El conocimiento del lenguaje (Knowledge of language), la idea de que langue es un sistema de reglas internalizadas. Para Newmeyer sin embargo, esa no era la intención de Chomsky. La atribución aparente no era más que el uso de un dispositivo retórico de un tipo que Chomsky había aprovechado varias veces en publicaciones anteriores. En síntesis, Newmeyer sostiene que, correctamente interpretada, la cita no encarna de hecho una evaluación cambiada de Saussure por parte de Chomsky.

El séptimo artículo, ampliando lo postulado en el artículo anterior y titulado precisamente Saussure and Chomsky Langue and I-language (Naoki Araki) contrasta los argumentos de los dos lingüistas alrededor de siete aspectos del lenguaje identificando además las diferencias entre ellos. Para tal efecto, se toman como base las nociones de langue (Saussure) e I-language (Chomsky): 1) cómo surgen los conceptos, 2) cómo aparecen los fonemas, 3) cómo cambian las lenguas 4) a dónde pertenece la sintaxis, 5) si el lenguaje es social o biológico, 6) cuáles son las partes del discurso, y 7) si las lenguas están delimitadas o no. Al hacerlo, se comprenderá que de qué maneras los dos lingüistas tienen ideas comunes o diferentes sobre el lenguaje.

En el octavo artículo titulado Ferdinand de Saussure y el Curso de Lingüística general (Xavier Laborda) el autor destaca, al margen del relato historiográfico de la lingüística, la preocupación central de Saussure, esto es montrer au linguiste ce qu'il fait! Critica en cierta forma la manera como se ha reconstituido el paradigma saussureano a partir de la reconstrucción del proceso editorial del Curso y plantea dos ideas frente a la 'vigencia' del paradigma saussureano a cien años de la aparición del Curso. La primera es que no hay tal paradigma saussureano, se niega su existencia y la segunda es que a pesar del peso destacable del paradigma saussureano en la lingüística actual, mantiene una vigencia relativa. Y queda planteada una suerte de paradoja cuando se descubre que además de tener que practicar la lingüística, el lingüista se empeña en teorizar sobre el proceso histórico de su disciplina (si no anduvieran por las ramas de la historia, la lingüística avanzaría más) desaprovechando la principal enseñanza de Saussure: "no basta con hacer, sino que también se debe pensar sobre qué y cómo se hace".

El último artículo, Ce que les théories du discours doivent à Saussure (Pierre-Yves Testenoire) hace un seguimiento a la presencia de postulados y conceptos saussureanos en las diferentes teorías contemporáneas sobre el discurso. Le interesa particularmente al autor describir las modalidades de la evolución de la concepción saussureana de 'discurso', previa lectura de su textos, pues en el lapso de treinta años, en el discurso sobre el discurso se muestra a dos Saussure: un primero que ignora la noción de discurso y discursividad y un segundo que la implementa y la toma como centro de su trabajo.

La tercera sección de este dossier, 'traducciones', presenta inicialmente la reseña que la profesora Créola Băltărețu Thénault le hace al reciente libro del profesor Rastier, Saussure de ahora en adelante (2015). La reseña describe en detalle el proyecto y compromiso rasterianos, esto es la relectura de Saussure con nuevos ojos: François Rastier nos invita así a imaginar "Saussure de ahora en adelante", sobre todo leyendo y releyendo los inéditos de 1996 y publicados en 2002, ya que estos textos pertenecen perfectamente "a nuestro siglo": "A nosotros nos corresponde leerlos, interpretarlos, valorar su alcance con respecto a la fecha de su redacción (verosímilmente la década de 1890) como en relación al corpus saussureano, a la historia misma 
del saussurismo y al porvenir de la lingüística" (cf. Introducción, p. 10).

Le sigue un artículo polémico y esclarecedor de la pluma del profesor alemán Jürgen Trabant titulado ¿Hay que proteger a Saussure de sus seguidores? Notas item sobre la etimología saussureana. Mientras que para autores como Rastier, Bouquet y Mejía es importante, incluso imperativo volver a Saussure, acercarse a él, redescubrirlo, para Trabant por el contrario resulta poco práctico volver a Saussure dudando incluso de su utilidad para la lingüística actual: la lingüistica actual se encuentra en planetas infinitamente alejados delagalaxiallamada "Saussure". Prácticamente no se interesa en nada de lo que "Saussure" representa (con excepción posiblemente del problema de la reacción entre sincronía y diacronía). Critica además los intentos por rescatar la imagen del verdadero Saussure (quien no escribió el CLG) a través de la exhumación filológica, como un ensayo de actualización: El problema, con este ensayo de actualización, es que no es el Saussure conocido el que él le vuelve a proponer a la lingüística sino un Saussure nuevo, un Saussure que está aún más alejado de la lingüística actual que el antiguo Saussure, sobre el cual la lingüística ha mantenido, al menos aún, algunos prejuicios ("the arbitrary Saussurean sign") - al igual que con Humboldt además, cuando la lingüística cree que fue el inventor de la tipología; y a lo largo del artículo insiste en la problemática de una lectura etimológica/filológica de los manuscritos de Saussure, en particular los Ecrits sin la referencia al Curso, sin apoyarse o compararse con el Curso ya que, es lo inevitable, la versión deconstructiva de Saussure, el Saussure auténtico, etimológico, verdadero, no tendrá la fuerza para eliminar el poder del Gran Clásico, por más usurpado o falso que sea dicho poder. El Saussure «auténtico» está condenado trágicamente a seguir siendo el bufón del Curso.

De todos los trabajos que hacen parte de este dossier, solo cuatro de ellos son inéditos. Le Cours de linguistique générale et l'histoire du saussurisme (Anne-Gaëlle Toutain) Un bon exercice de lecture $d u$ Cours de linguistique générale (Claire Forel), Les cours de linguistique générale de Ferdinand de
Saussure, un projet de Paul-F. Regard et Antoine Meillet y Lingüistica general de Ferdinand de Saussure. El primer curso. Introducción. (Claudia Mejía). Los dos primeros textos fueron escritos especialmente para este número. Como respuesta a la convocatoria, los autores de los demás artículos propusieron trabajos ya publicados y fueron modificados para adecuarlos a "los requerimientos" de la revista. Agradecemos a los autores su valiosa participación.

\section{Referencias}

Mahecha, M.A. (2003). [Reseña del libro «Ecrits de Linguistique générale» de Ferdinand de Saussure. Paris, Gallimard], Forma y Función 16, pp. 310-323.

Rastier, F. (2015). Saussure au futur. Paris: Les Belles Lettres.

Rastier, F. (2012). Lire les textes de Saussure. Langages 185. Paris: Larousse.

Calvet, L.-J. (1975). Pour et contre Saussure. Paris: Payot.

Gadet, F. (1990). Saussure. Une science de la langue. Paris : PUF.

De Lemos, et al. (2003). Le Saussurisme en Amérique Latine au XXe siècle. Cahiers Ferdinand de Saussure, No. 56, pp. 165-176.

Sofia, E. (2016). Modos de recepción y difusión del Curso de lingüística general de F. de Saussure en América Latina. Presentación del dossier, Signo y Seña 30, pp. 1-2

Nethol, A. (1977). Ferdinand de Saussure. Fuentes manuscritas y estudios críticos. México: Siglo XXI editores.

Peña, H. (2016). Trabajo de la lengua /Trabajo de sentido: la lección de Alcira Saavedra sobre Ferdinand de Saussure. Signo y Seña 30, pp. 37-52. 